\title{
Importance of head axes in perception of cutaneous patterns drawn on vertical body surfaces
}

\author{
KAORU SEKIYAMA \\ Kanazawa University, Kanazawa, Japan
}

\begin{abstract}
The "disembodied eye" phenomenon (Corcoran, 1977), that is, the observation that a cutaneous (tactile) pattern is perceived as right-left reversed or not, depending on whether it is presented on the forehead or the back of the head, was extended by incorporating a top-bottom axis into a model of the frame of reference in cutaneous-pattern perception. In two experiments, 21 and 15 subjects were asked to report the letter perceived when one of four letters (p, q, b, or d) was tactually presented. The sites studied were vertical body surfaces (Experiment 1 ) and hands alongside the body (Experiment 2). The positions for the presentations varied according to the height (head, shoulder, waist, thigh, and calf levels) and the orientation of the surface: forward-, backward-, and side-facing. Although the results for the head and back surfaces supported the notion of a "disembodied eye" behind the individual, other frames were needed: On the forward-facing surfaces below the waist, the prevailing perception was $180^{\circ}$ rotated, as if the subjects were looking at the surface by bending forward. An additional frame of reference was introduced for the forwardfacing surfaces in lower positions and was described as head axes projected onto body surfaces within the possibility of actual body movements.
\end{abstract}

In cutaneous (tactile) pattern perception, there is a phenomenon that can be called the "disembodied eye" phenomenon (Corcoran, 1977). When a letter $p$ is tactually presented on subjects' forehead, most of the subjects perceive it as lowercase $\mathrm{q}$, which is a right-left reversal of objective tactile stimulation. If, however, the stimulation is given on the back of the head, such reversed perception does not occur. There has been sufficient data to establish this phenomenon, that is, reversed perception on front surfaces, especially on the forehead (Allen \& Rudy, 1970; Corcoran, 1977; Duke, 1966; Holmes, Roeckelein, \& Olmstead, 1968; Krech \& Crutchfield, 1958; Natsoulas \& Dubanoski, 1964; Parsons \& Shimojo, 1987; Sekiyama, 1981). In this paper, the disembodied-eye phenomenon is defined as the perception of a cutaneous pattern as reversed or objective, depending on whether it is presented on the forehead or on the back of the head.

Several researchers have proposed similar accounts of this phenomenon. Corcoran (1977) assumed that there is a "disembodied eye" that is located behind one's head. According to his concept of the "disembodied eye," a

This research was supported by the Japanese Ministry of Education, Science, and Culture Grant-in-Aid for Scientific Research No. 60790005 to the author. It was carried out while the author was at Osaka City University. I thank Lawrence Parsons and Thomas Hofmann for their useful comments on earlier drafts of this article. I also wish to thank Fukuko Inoue and Kiyo Yoshikawa for their invaluable assistance in running the experiments. Requests for reprints and other correspondence should be addressed to Kaoru Sekiyama, Department of Psychology, Kanazawa University, Kakuma-machi, Kanazawa, 920-11 Japan. cutaneous pattern presented on the forehead is "seen"1 in a penetrating way, since the "disembodied eye" is on the opposite side of the surface. Duke (1966) proposed the frontal plane hypothesis, according to which symbols drawn upon anterior and posterior surfaces of the body are perceived as if they were projected forward onto a surface in front of the subject. Krech and Crutchfield (1958) interpreted the reversed perception on the forehead in terms of the internal locus of the perceiver.

The common idea for these three accounts is that we perceive a cutaneous pattern as if we "saw" our body surfaces by a sort of "line of sight" that is directed forward. This phenomenon suggests an egocentric aspect of our space recognition. Its implication is helpful in understanding how the perceptual system integrates information from tactile receptors.

The implication of the disembodied-eye phenomenon becomes clearer when a cutaneous pattern is presented on the palm or back of the hand. Corcoran (1977) pointed out that when a letter $p$ was drawn on the forward-facing, head-level palm, the $p$ was perceived reversely as a letter $q$, whereas on the backward-facing palm, a $p$ was objectively perceived as a $\mathbf{p}$ (Figure 1). This is sufficiently supported by the more recent data of Parsons and Shimojo (1987). Note that, in both forward-facing and backwardfacing conditions, information from tactile receptors on the palm is identical. The only difference is the orientation of the palm. This means that cutaneous-pattern perception is not a direct projection of the stimulation to the surface. Rather, it requires a more psychological frame of reference that is related to the body-relative orienta- 


\section{STIMULATION}

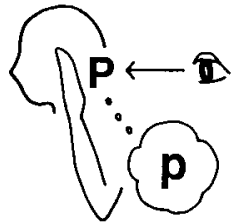

PALM FACING BACKWARD

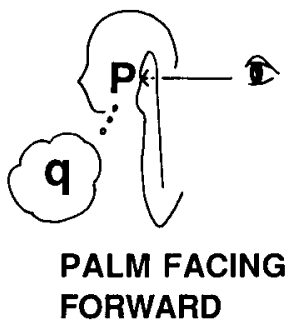

Figure 1. The "disembodied eye" phenomenon on hands. Whether the subject perceives this pattern as a letter $p$ or a letter $q$ depends on the direction of the palm.

tion of the surface. This frame of reference is the basis not only for perceiving cutaneous patterns but also for motor production of patterns (Shimojo, 1981; Shimojo, Sasaki, Parsons, \& Torii, 1989).

In the field of visual space perception, there has been an analogous question of how the perceptual system integrates retinal information that undergoes transformation based on movements of the perceiver's eyes or body and of external objects. Koffka (1935) was probably the first to emphasize the role played by the frame of reference, which integrates unstable sensory input. Koffka's terminology of the "framework" originally implied visual frames, such as those provided by walls, floors, and the horizon.

More recent researchers, however, noticed that, for perception and apparent orientation of shape, there are several axes of reference to which the perceiver can relate the orientation of shapes (e.g., Howard \& Templeton, 1966; Rock, 1973). Howard (1982) classified such frames into two groups: intrinsic and extrinsic axes of the object. For example, a square can look like a diamond, depending on the change of its intrinsic axes, that is, when it is rotated by $45^{\circ}$. Extrinsic axes, on the other hand, are outside the object and comprise three types: the visual frame, gravity, and the egocentric axes (retinal or other body axes of the perceiver). A tilting rectangle provides a visual frame that is incongruent with the gravity and egocentric axes. When subjects tilt their heads, or look backwards through their legs, the egocentric axes are inconsistent with gravity and the visual frame. A series of experiments by Rock (Rock, 1956; Rock \& Heimer, 1957) revealed that, to perceive unfamiliar shapes, gravity is often the most important axis and that the retinal axis is less important. Recently, this issue was dealt with in a more cognitive task such as mental rotation under head tilt (Corballis, Zbrodoff, \& Roldan, 1976; Sekiyama, 1982).

Compared with the advances in the field of visual space perception, there have not been enough studies on frame of reference in tactile perception. Even in visual perception, nonvisual factors such as gravity and the proprioceptive factor play a role. This shows multimodal or nonmodality specific aspects of space perception. Thus, to investigate the frame of reference in tactile perception would be of some interest. The disembodied-eye phenomenon may provide a means to solve this problem.

The perception of a cutaneous character on the skin is regarded as a process of assigning the top-bottom and right-left coordinates to it (Oldfield \& Phillips, 1983). We may assign these spatial coordinates in relationship to any of three sets of axes. One is the set of egocentric axes, in which the top is the perceiver's head and the left is his/her left-hand side. Second, we may refer to gravity. Third, the axes of the object itself can be decisive for familiar objects. The disembodied-eye phenomenon suggests that the egocentric axes are more important as a frame of reference than the other axes in tactile pattern perception.

Earlier research in the 1960s has shown that reversed perception consistently occurs on the forehead (Allen \& Rudy, 1970; Duke, 1966; Holmes et al., 1968; Natsoulas \& Dubanoski, 1964). Only a few recent studies have examined this phenomenon for various body parts other than the head (Corcoran, 1977; Parsons \& Shimojo, 1987; Sekiyama, 1981). Compared with the clear results for the head, the data for the body, hands, and the legs are not so simple, suggesting other factors of reference. Further data should be accumulated.

Parsons and Shimojo (1987) first explored this phenomenon in detail, manipulating the factors of body part, orientation of surface, body-relative position of surface, and body configuration. Although their results for front and back surfaces of the head were consistent with the disembodied-eye explanation, the results for the other parts of the body or hands were complicated and required several specific explanatory rules. They tried to explain those results by proposing some frames of reference associated with the head, the upper body, and the hands.

Corcoran (1977) and Sekiyama (1981) obtained results inconsistent with the disembodied-eye explanation, especially when stimuli were drawn on the legs. That is, $180^{\circ}$ rotated responses, such as a response " $\mathrm{d}$ " for the stimulus $p$, occur on the front surface of the thigh, as if the subject was looking at the pattern by bending. Corcoran (1977) proposed a "pretend look" hypothesis to explain this kind of response. No such responses were found on vertical surfaces of the head or vertically positioned hands at shoulder level. In line with these facts, Parsons and Shimojo (1987) obtained results that indicated that, on forward-facing surfaces of trunk, arm, thigh, and hand at thigh level, most of the responses were matching "experimenter-defined handedness" (" $180^{\circ}$ rotated" and "objective" responses are combined), whereas the forehead showed right-left reversed responses. These results suggest that (1) the perceived top-bottom of a cutaneous pattern is influenced by the body-relative height of the surface and that (2) the frame of reference in cutaneouspattern perception may have a visuomotor aspect such as "pretend look."

The purpose of this paper is to examine the top-bottom axis assignment in the light of the pretend-look hypothesis. The advantage of this hypothesis is that it gives us 
a very intuitive understanding of the frame of reference. In addition, this hypothesis describes well a dynamic aspect of human recognition. Although Corcoran proposed the pretend-look hypothesis to account for his informal observations for the thigh, in fact, he did not report a formal experiment for it. Therefore, systematic experiments were required to examine this hypothesis. Our questions are: (1) to what extent is this hypothesis valid? and (2) to which part of the body does this hypothesis fit?

To examine these questions, there was a methodological problem to be solved. In the studies concerning the disembodied-eye phenomenon, the traditional manner of data classification was not appropriate to deal with the pretend-look hypothesis. In most of the previous research, the responses have been classified into the binary categories according to whether they were viewed from the experimenter's viewpoint or the subject's viewpoint. Taking Parsons and Shimojo's experiments (1987), for example, both " $p$ " and " $\mathrm{d}$ " responses for the stimulus $p$ were regarded as "experimenter-defined handedness" and " $q$ " and " $b$ " responses were regarded as "mirror reversed." All their systematic analyses of the data were based on the binary categorization. This same classification has been taken in most studies (Allen \& Rudy, 1970; Duke, 1966; Holmes et al., 1968; Natsoulas \& Dubanoski, 1964). Logically however, there are four possible responses, that is, “objective" (stimulus $p \rightarrow$ response “ $p$ "), "right-left reversed" ( $p \rightarrow$ " 'q"), "up-down inverted" ( $p \rightarrow$ “ $b$ "), and "rotated by $180^{\circ}$ " ( $p \rightarrow$ " $\mathrm{d}$ "). Data recorded in the binary categorization can tell us only how the subjects assigned the right-left axis to the pattern. As long as only the head was studied, it was enough to observe the right-left axis assignment, because subjects always perceived the top of the cutaneous pattern as consistent with the top of their head. However, the top-bottom axis assignment does vary in the lower part of the body (Corcoran, 1977; Sekiyama, 1981), as described above. When it comes to dealing with the pretend-look hypothesis, the top-bottom axis assignment that is missing in the binary categorization is critical.

This study investigated the effect of the height of the stimulated surface on the top-bottom axis assignment through systematic experiments in which subjects' responses were categorized into four types. In Experiment 1, cutaneous patterns were presented on various body regions of various heights. In Experiment 2, the patterns were drawn on the palm and back of the hands, with the hands positioned at various heights.

\section{EXPERIMENT 1}

The notion of the "disembodied eye" located behind one's head predicts right-left reversed response on the front surfaces and objective response on the back surfaces. On the thigh and other low-level surfaces, however, results not fitting this prediction were found in Corcoran (1977), Parsons and Shimojo (1987), and Sekiyama (1981). As described above, these results suggested that the perceived top-bottom of a cutaneous pattern is influenced by the height of the surface. In the present experiments, the top-bottom axis assignment was investigated as a function of the height of the surface. Experiment 1 studied vertical body surfaces in various heights. Although Parsons and Shimojo (1987) also studied these surfaces, their binary categorization missed information concerning subjects' top-bottom axis assignment.

\section{Method}

Subjects. Twenty-one students majoring in psychology at Osaka City University served as subjects.

Stimuli. The stimulus patterns were lowercase letters $p, q, b$, and $d$. The experimenter drew these letters at least $5 \mathrm{~cm}$ high on the subject's skin with a smooth rounded metal instrument (a pen cap). Each letter was drawn in one stroke, beginning from the end of the vertical line so that none of the letters were written in their conventional ways.

Procedure. The subjects stood in a natural posture with their eyes closed. The experimenter drew one of the four letters on their skin. After each drawing, the subjects were asked to report the character perceived. Oral responses of their instantaneous impressions were elicited, although fast responses were not required. Repeated presentation of a stimulus was permitted when the subject failed to get a clear impression the first time. Instructions emphasized reporting what the subject perceived, not what the experimenter intended to draw. The response category was recorded as follows. When the experimenter drew a stimulus $p$, a p-response was categorized as objective and a q-response as right-left reversed. Likewise, a b-response was classified as up-down inverted and a d-response as rotated by $180^{\circ}$.

Design. The experimental variables were the height and the orientation of the surface (Figure 2). The heights were at head, chest, waist, thigh, and calf levels. At each level, stimuli were presented on four surfaces: front, back, right, and left surfaces, giving 20 experimental conditions: 5 (heights) $\times 4$ (orientations). As for the thigh and calf levels, the front and back observations were repeated for both the right and left legs. Each subject participated in all the conditions. The order of conditions was random and, in each condition, the four letters were presented at random.

\section{Results and Discussion}

In Figure 3, each bar shows the frequency of the four response categories in each condition.

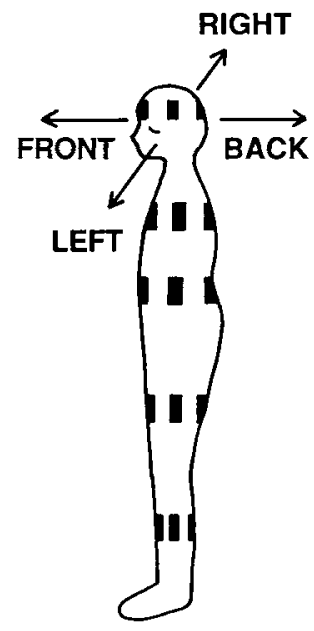

Figure 2. The experimental conditions in Experiment 1. The experimental variables were the height and direction of the surface. 


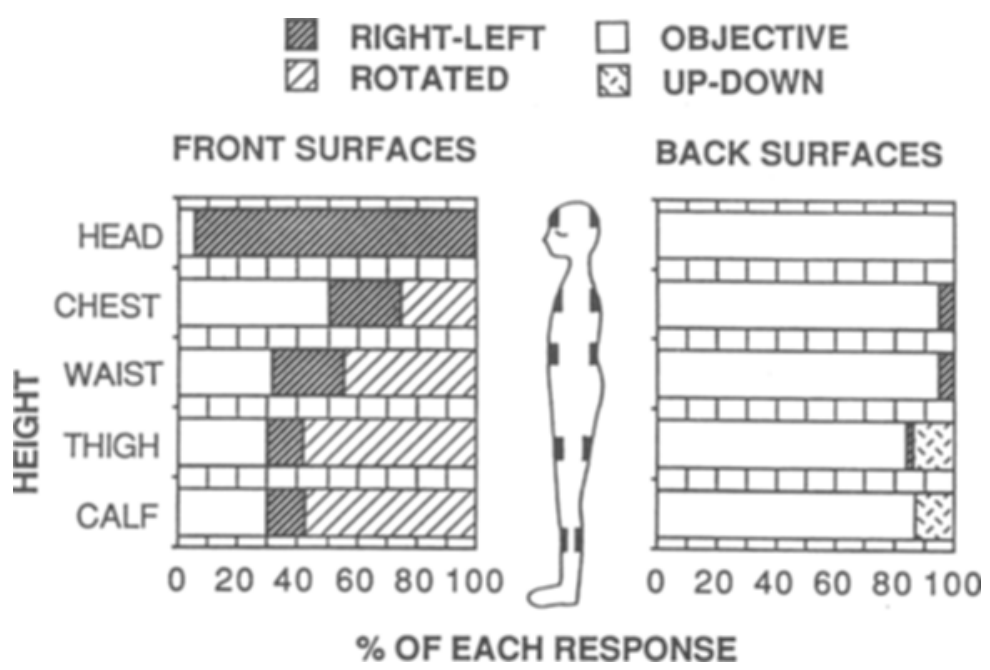

Figure 3. Mean rates of each response in each height on the front and back surfaces of the body.

Results for the front and back surfaces. Figure 3 shows the results for the front and back surfaces. The notion of the "disembodied eye" predicts objective response on the back surfaces and right-left reversal on the front surfaces. The results that were obtained only partially supported this prediction. The response depended not only on the front-back orientation but also on the height of the surface. The effect of height is remarkable, especially on the front surfaces, in which the predicted response of right-left reversal is minor below the neck. At the head level, that is, on the forehead, $96 \%$ of the responses were right-left reversal, as predicted. On the chest, however, the right-left reversal reduces to $25 \%$, and the most frequent response is objective (50\%). In the waist, thigh, and calf levels of the front surfaces, the right-left reversal is also minor $(25 \%, 12 \%$, and $13 \%$, respectively) and the most frequent response is rotated $(45 \%, 58 \%$, and $57 \%$, respectively). Thus, below the neck, the prevailing responses are rotated and objective. The frequency of the rotated response is a function of the height of the stimulated surface-the lower the level, the more frequent the rotated response.

On the back surfaces, most of the responses are objective, as predicted by the disembodied-eye account. In the thigh and calf levels, however, this objective response decreases and up-down inversion occurs about $15 \%$ of the time.

The likelihood ratio tests ${ }^{2}$ within both the front and back surface conditions confirmed that the effect of the surface height was statistically significant: For each of the front and back surfaces, the likelihood ratio chi-squares were 218.065 and 54.990, respectively $(d f=8, p<.001$, in each case). The effect of the height was also tested within each adjacent height pair, as shown in Table 1: For the front surfaces, significant differences were found in the head-versus chest-level and chest-versus waist-level pairs. For the back surfaces, it was in the head-versus chest-level and waist- versus thigh-level pairs that the likelihood ratio tests revealed significant differences.

Summarizing the data, it is only for the head-level condition that the results could be accounted for by a notion of a "disembodied eye." For the lower surfaces,

Table 1

Percentage of Each Response in Each Condition and the Effect of the Surface Height in Each Adjacent Pair (Body)

\begin{tabular}{|c|c|c|c|c|c|c|c|}
\hline \multirow[b]{2}{*}{ Conditions } & \multicolumn{4}{|c|}{ Response Category } & \multirow{2}{*}{$\begin{array}{c}\text { Likelihood } \\
\text { Ratio } \\
\text { Chi-Square }\end{array}$} & \multirow[b]{2}{*}{$d f$} & \multirow[b]{2}{*}{$p$} \\
\hline & OBJ & L-R & ROT & U-D & & & \\
\hline \multicolumn{8}{|c|}{ Front } \\
\hline $\begin{array}{l}\text { Head } \\
\text { Chest } \\
\text { Waist } \\
\text { Thigh } \\
\text { Calf }\end{array}$ & $\begin{array}{r}4.8 \\
50.0 \\
30.9 \\
29.8 \\
30.4\end{array}$ & $\begin{array}{l}95.2 \\
25.0 \\
25.0 \\
11.9 \\
12.5\end{array}$ & $\begin{array}{c}0 \\
25.0 \\
44.1 \\
58.3 \\
57.1\end{array}$ & $\begin{array}{l}0 \\
0 \\
0 \\
0 \\
0\end{array}$ & $\begin{array}{r}102.46 \\
8.27 \\
5.69 \\
0.03\end{array}$ & $\begin{array}{l}2 \\
2 \\
2 \\
2\end{array}$ & $\begin{array}{l}.000 \\
.016 \\
.058 \\
.986\end{array}$ \\
\hline \multicolumn{8}{|c|}{ Back } \\
\hline $\begin{array}{l}\text { Head } \\
\text { Chest } \\
\text { Waist } \\
\text { Thigh } \\
\text { Calf }\end{array}$ & $\begin{array}{r}100 \\
95.2 \\
95.2 \\
83.3 \\
86.9\end{array}$ & $\begin{array}{c}0 \\
4.8 \\
4.8 \\
2.4 \\
0\end{array}$ & $\begin{array}{l}0 \\
0 \\
0 \\
0 \\
0\end{array}$ & $\begin{array}{c}0 \\
0 \\
0 \\
14.3 \\
13.1\end{array}$ & $\begin{array}{c}5.64 \\
- \\
17.98 \\
2.88\end{array}$ & $\begin{array}{l}\frac{2}{2} \\
2 \\
2\end{array}$ & $\begin{array}{l}.018 \\
- \\
.000 \\
.237\end{array}$ \\
\hline \multicolumn{8}{|c|}{ Right } \\
\hline $\begin{array}{l}\text { Head } \\
\text { Chest } \\
\text { Waist } \\
\text { Thigh } \\
\text { Calf }\end{array}$ & $\begin{array}{l}81.0 \\
56.0 \\
45.2 \\
62.5 \\
53.6\end{array}$ & $\begin{array}{r}19.0 \\
27.4 \\
21.4 \\
3.6 \\
9.5\end{array}$ & $\begin{array}{c}0 \\
16.7 \\
33.3 \\
31.5 \\
36.9\end{array}$ & $\begin{array}{c}0 \\
0 \\
0 \\
2.4 \\
0\end{array}$ & $\begin{array}{r}24.52 \\
6.32 \\
17.03 \\
0.95\end{array}$ & $\begin{array}{l}2 \\
2 \\
2 \\
2\end{array}$ & $\begin{array}{l}.000 \\
.042 \\
.001 \\
.621\end{array}$ \\
\hline \multicolumn{8}{|c|}{ Left } \\
\hline $\begin{array}{l}\text { Head } \\
\text { Chest } \\
\text { Waist } \\
\text { Thigh } \\
\text { Calf }\end{array}$ & $\begin{array}{l}81.0 \\
65.5 \\
47.6 \\
66.7 \\
71.4 \\
\end{array}$ & $\begin{array}{c}19.0 \\
19.0 \\
20.2 \\
0 \\
6.0\end{array}$ & $\begin{array}{c}0 \\
15.5 \\
32.1 \\
33.3 \\
22.6 \\
\end{array}$ & $\begin{array}{l}0 \\
0 \\
0 \\
0 \\
0 \\
\end{array}$ & $\begin{array}{r}19.40 \\
7.41 \\
26.26 \\
8.80\end{array}$ & $\begin{array}{l}2 \\
2 \\
2 \\
2\end{array}$ & $\begin{array}{l}.000 \\
.025 \\
.000 \\
.012\end{array}$ \\
\hline
\end{tabular}

Note-OBJ = objective; $\mathrm{L}-\mathrm{R}=$ left-right; $\mathrm{ROT}=$ rotated $\mathrm{U}-\mathrm{D}=$ up-down. 


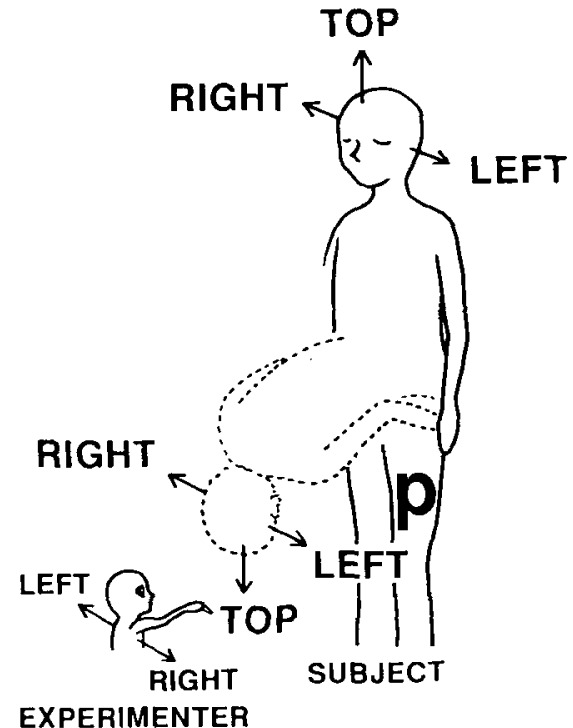

Figure 4. The head axes in a bending position as the basis for the rotated response on lower-front surfaces.

the disembodied-eye explanation is inadequate--we need another explanation. We must consider rotation and updown inversion, which were observed only in the lower conditions.

A bending-forward frame. We can interpret the rotated response as an outcome of the Corcoran's pretendlook explanation. In Figure 4, the experimenter is writing a $\mathrm{p}$ on the front surface of the subject's thigh. Now, if the subject were to bend forward to look at the letter, he would probably report that he sees a $d$ rather than a p. The reason is as follows: When the subject is bending over looking at his thigh, he perceives the vertical line of the $\mathrm{p}$ as pointing up (it is pointing down for the experimenter), because it points in the same direction as the top of his head. The curve of the letter is perceived on the left of that line, since it is on his left-hand side, whereas it is on the experimenter's right-hand side. As a result, perceived orientation is a $180^{\circ}$ rotation from the experimenter's orientation (rotation by $180^{\circ}$ is equal to the combination of the up-down inversion and the right-left reversal). This kind of "head response" was actually reported in visual perception (Thouless, 1947, cited in Howard \& Templeton, 1966). When Thouless's subjects had to look backwards through their legs, they saw one of two faces in an ambiguous picture according to their egocentric upright rather than the gravitational upright. If we hypothesize a "pretend look" in this manner, that is, a bending-forward frame in the processes of perceiving cutaneous patterns, we can easily understand the rotated response.

The same explanation is also valid for the up-down inversion on the back surfaces. In Figure 5, we assume that the bending-forward frame is projected onto the back surface of the subject's thigh, penetrating the thigh. Based on this projected frame, the vertical line of the $p$ is per- ceived as pointing up, because it points in the same direction as the top of his head. The curve of the $p$ is perceived on the right of the vertical line, since it is on his righthand side. In this way, a p on the back of the thigh is perceived as $a b$. This is an up-down inversion of the experimenter's orientation.

Results for the right and left surfaces. Figure 6 shows the results for the right and left surfaces. The results for the right and left surfaces are almost the same (likelihood ratio tests within each height showed significant right-left differences in only one [thigh] height level). At the head level, $80 \%$ of the responses were objective and $20 \%$ were right-left reversed. Compared with the front and back surfaces of the head (Figure 3), these are intermediate responses between those for the forehead and the back of the head, although they are closer to the results for the back of the head. Below the neck, again there is a rotated response, for which the disembodied-eye explanation is inadequate. Figure 6 shows that the rotated response occurred frequently on the lower part of the right and left surfaces, as well as on the front surfaces. This can be again explained by mental bending.

The effects of height within the left and right surfaces were statistically significant: the likelihood ratio chisquares were 86.789 and 85.637 , respectively $(d f=8$, $p<.001$, in each case). As for the effect of the height within each adjacent height pair (Table 1), significant differences were found in all but one pair (thigh- vs. calflevel pair in the right surface).

Constraints of the bending-forward frame. The results for the lower levels were reasonably accounted for by the pretend-look hypothesis. However, this can be more accurately described as a bending-forward frame. To clarify the nature of this frame of reference, the following facts should be considered: (1) The rotated response did not occur on the head-level surfaces, whereas it did

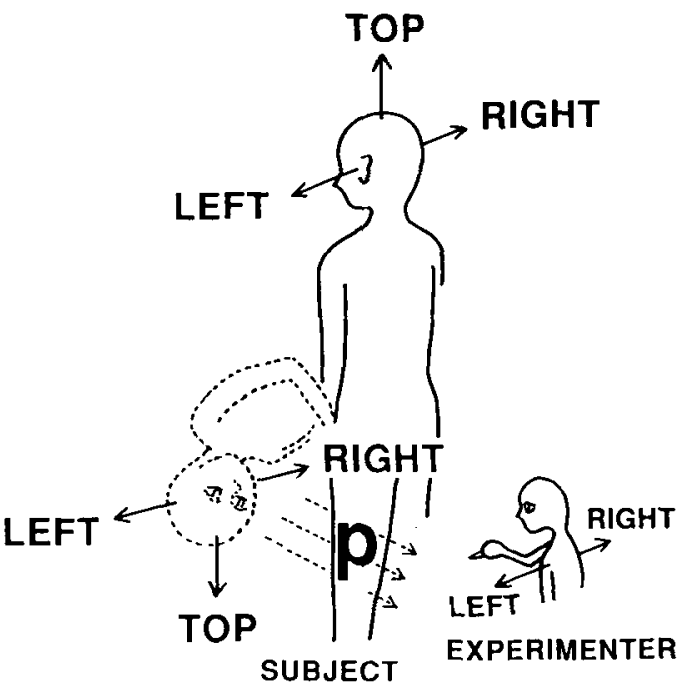

Figure 5. Projection of the head axes onto the back surface of the thigh, inducing the up-down inverted perception. 


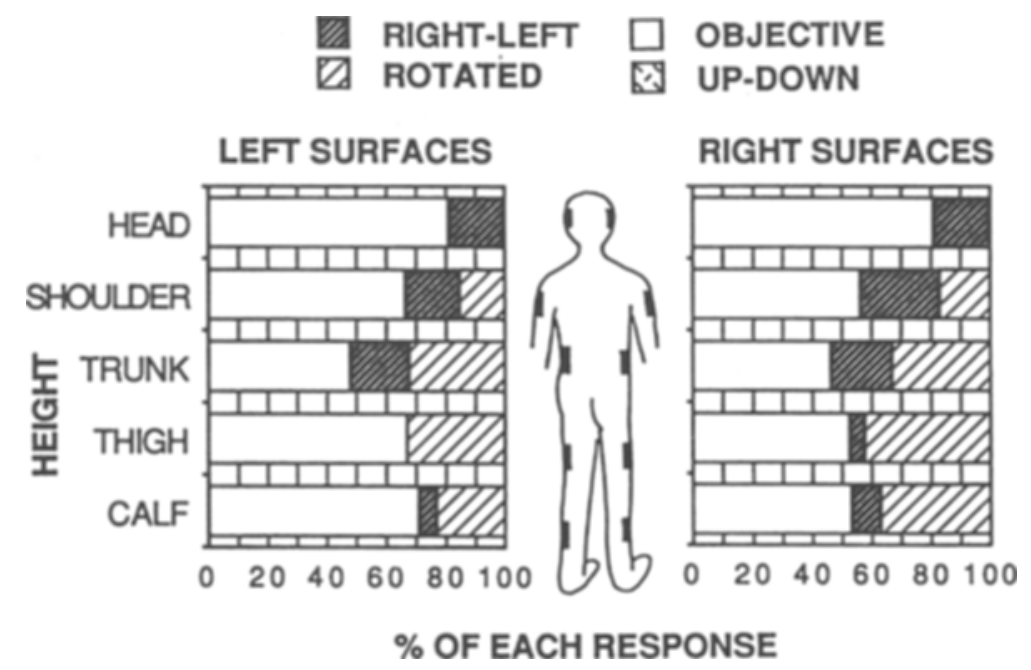

Figure 6. Mean rates of each response at each height on the right and left surfaces of the body.

occur to some extent below the neck; (2) the rotated response on the front surfaces increased as a function of its distance from the head; and (3) although most of the responses for the back surfaces were objective, some responses in the thigh and calf levels were up-down inverted. These three facts follow quite naturally from our bending-forward frame. Actual bending enables us to see certain parts of ourselves: the front and lateral surfaces below the neck. No matter how we move the head, the head itself cannot be viewed directly. Neither can we view back surfaces. In line with these physical constraints, the rotated response did not occur either on the head or on the back surfaces. On the other hand, the rotated response on the front surface increased as a function of the distance from the head. This corresponds to another physical property: the lower the surface, the easier it is to see by bending forward. These facts suggest that the physical properties of the body are represented in the frames of reference. That is, these frames of reference are associated with the nature of the body's movability. The application of a frame is limited in accordance with the physical constraints.

Corcoran's (1977) pretend-look hypothesis included physically impossible movements such as bending the neck $360^{\circ}$ backward to enable an individual to view back surfaces directly. It is implausible to assume such movements. Moreover, the back of the head cannot be seen directly no matter how far backward the neck is bent. Thus, the objective responses on back surfaces cannot be accounted for by the pretend-look hypothesis. Rather, the up-down inverted response obtained in Experiment 1 suggests that a bending-forward frame is sometimes used to perceive a stimulus presented on the back of thighs and calves.

Individual differences. Analysis and discussion of the results above were based on group data. There were, in fact, three types of subjects. Figure 7 shows individual data for front, back, and right surfaces. Each cell depicts frequencies of the four response categories with their four responses. Taking the data for front surfaces (Figure 7a), for example, Subject 1 (S.S.) gave the right-left reversed response in all the four trials for the forehead (head level), whereas all his trials for the chest (chest level) resulted in rotated responses. Looking at the head-level results for front and back surfaces, all but one of the subjects show right-left reversed responses on the forehead (Figure 7a) and objective responses on the back of the head (Figure 7b), indicating stability of the disembodied-eye phenomenon.

Individual differences occurred in the conditions below the neck. Those individual differences can be classified into three groups: For front surfaces (Figure 7a), (1) Subjects 1-14 show rotated responses, suggesting a bendingforward frame; (2) Subjects 15-17 show right-left reversed responses, accounted for by the "disembodied eye" positioned at the back of the subject; and (3) Subjects 18-21 show objective responses, indicating an objective frame of reference in which a viewpoint is positioned wherever necessary around the body to directly face the stimulated surface. For back surfaces (Figure 7b), some subjects in Group 1 (Subjects 3, 4, 8, 10,11) show up-down inverted responses, also suggesting bending-forward axes with piercing vision. In the other subjects, most of the responses for the back surfaces were objective, showing the stable role of the "disembodied eye" for the back surfaces.

These results illustrate that individual differences come from the subjects' choice from among the three frames of reference: (1) the bending-forward frame, (2) the "disembodied eye," and (3) an objective viewpoint. The choices do not vary much from one trial to another. This is also true for the results for left and right surfaces (Figure 7c). In any event, we should not ignore these individual differences, which have not received significant attention in the published literature. They may be related 
FRONT SURFACES

(a) height of the surface
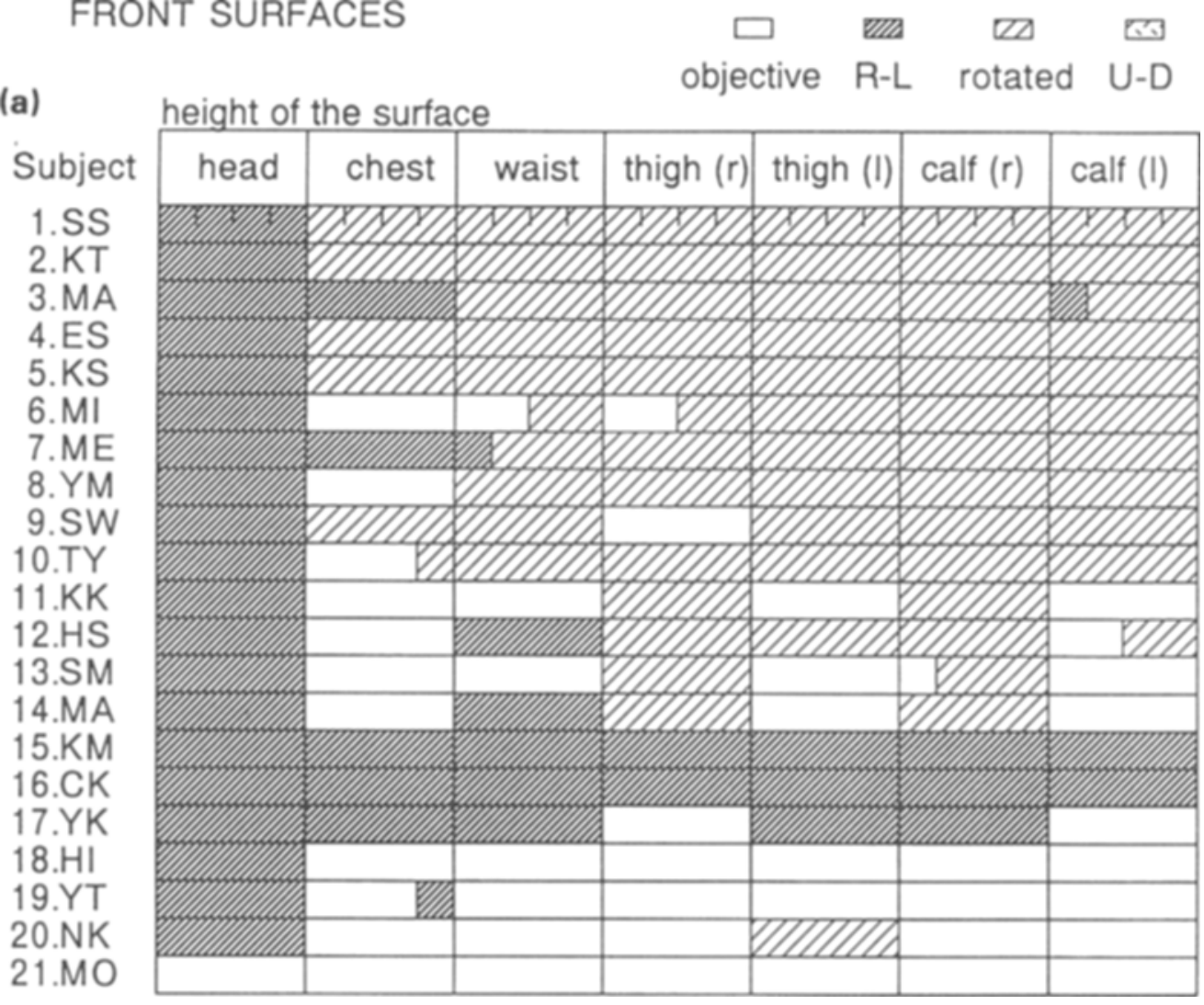

BACK SURFACES

口 ש ए ए

(b) height of the surface objective R-L rotated U-D

\begin{tabular}{|c|c|c|c|c|c|c|c|}
\hline Subject & head & chest & waist & thigh $(r)$ & thigh (I) & calf $(r)$ & calf (I) \\
\hline \multirow{2}{*}{\multicolumn{8}{|c|}{$\begin{array}{l}\text { 1.SS } \\
2 . \mathrm{KT}\end{array}$}} \\
\hline & & & & & & & \\
\hline \multirow{2}{*}{\multicolumn{8}{|c|}{$\begin{array}{l}\text { 3.MA } \\
\text { 4.ES }\end{array}$}} \\
\hline & & & & & & & \\
\hline \multicolumn{8}{|l|}{$5 . \mathrm{KS}$} \\
\hline \multicolumn{8}{|l|}{ 6.MI } \\
\hline \multicolumn{8}{|l|}{$\begin{array}{l}\text { 7.ME } \\
\text { 8.YM }\end{array}$} \\
\hline \multicolumn{8}{|l|}{ 8.YM } \\
\hline \multicolumn{8}{|l|}{$\begin{array}{l}\text { 9.SW } \\
\text { 10TY }\end{array}$} \\
\hline \multicolumn{8}{|l|}{ 10.TY } \\
\hline \multicolumn{8}{|l|}{ 11.KK } \\
\hline \multirow{2}{*}{\multicolumn{8}{|c|}{$\begin{array}{l}\text { 12.HS } \\
13 . \mathrm{SM}\end{array}$}} \\
\hline & & & & & & & \\
\hline \multicolumn{8}{|l|}{ 14.MA } \\
\hline \multicolumn{8}{|l|}{ 15.KM } \\
\hline \multicolumn{8}{|l|}{ 16.CK } \\
\hline \multicolumn{8}{|l|}{ 17.YK } \\
\hline \multicolumn{8}{|l|}{ 18.HI } \\
\hline 19.YT & & & & & & & \\
\hline 20.NK & & & & & & & \\
\hline 21.MO & & & & & & & \\
\hline
\end{tabular}


RIGHT SURFACES

(c)

height of the surface

objective R-L rotated U-D

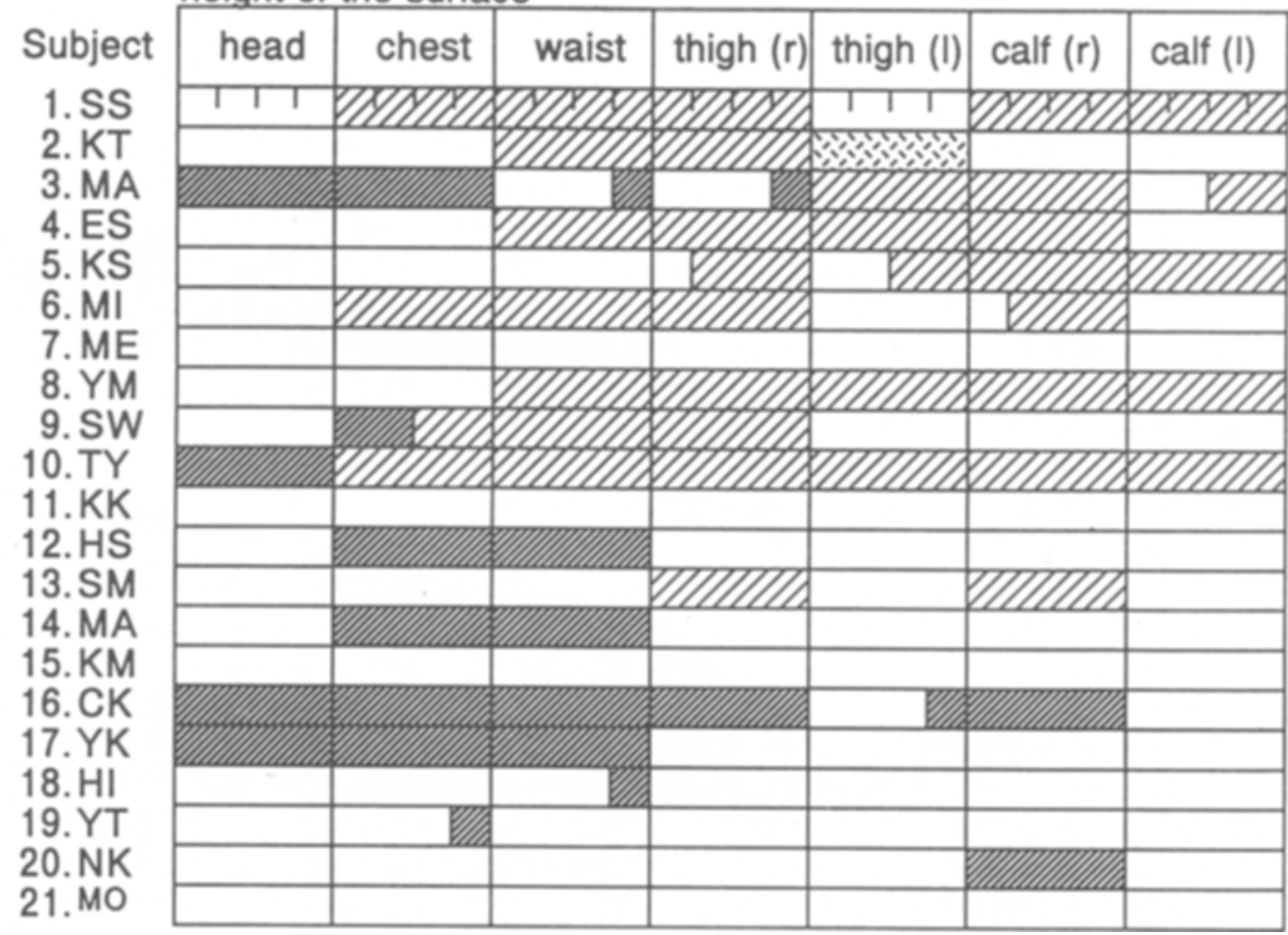

Figure 7 (page 487 and above). Individual data for front (a), back (b), and right (c), surfaces.

to the cognitive style of the individual, as studied in the rod-and-frame test and tilting-room-tilting-chair experiments (Witkin et al., 1954/1972).

A model of frames of reference. Parsons and Shimojo (1987) reported in their binary categorization analysis that the lower part of the vertical body surfaces yielded responses matching the experimenter-defined handedness (rotated and objective responses were combined). From their results, they concluded that the vertical body surfaces below the neck are perceived from an objective viewpoint. Our four-way categorization revealed, however, that responses for the front surfaces below the neck include both rotated and objective responses and that rotated responses vary as a function of the height of the stimulated surface. This rotated response suggests a bending-forward frame.

According to the results of Experiment 1, we should assume that there are at least two functionally different types of regions in the vertical body surfaces. One type includes the regions that we can never see directly, that is, the head and the back surfaces. The other type includes the regions that we can see directly by bending forward, that is, the lower part of the front and lateral surfaces. Experiment 1 revealed that the role of the "disembodied eye" is limited to the head-level surfaces and the back surfaces, whereas for most front surfaces below the neck, our results show that the subject tends to perceive cutane- ous patterns on these surfaces based on a bending-forward frame, although an objective viewpoint is also used. The notion of the bending-forward frame accounts for the rotated response on the front surfaces and up-down inversion on the back surfaces.

The lower regions showed greater variation in response compared with the head. This suggests that some frames of reference can simultaneously function for the lower surfaces, whereas the "disembodied eye" is the only frame of reference in the head-level.

\section{EXPERIMENT 2}

The question we ask in Experiment 2 is whether or not the bending-forward frame is also used for the hand surfaces. In Experiment 2, cutaneous patterns were always drawn on the palm and the back of the hands, and the hands were positioned at various heights alongside the body.

\section{Method}

Subjects. Fifteen students at Osaka City University served as subjects.

Procedure. The procedure was the same as that of Experiment 1.

Design. Experimental variables were the height and orientation of the hand (Figure 8). ${ }^{3}$ The hands were positioned at the overthe-head, top-of-the-head, head, waist, hip, and thigh levels, close to and parallel to the body. A ruler was used to fix the position of the hands. The orientations were forward-facing and backward- 


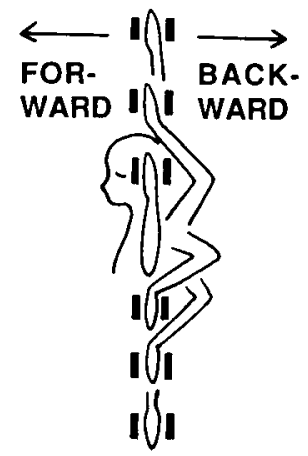

Figure 8. The experimental conditions in Experiment 2. The experimental variables were the height and direction of the hands.

facing. The total experimental conditions were 5 (height) $\times 2$ (direction) $\times 2$ (palm and back) $\times 2$ (right and left hands) $=40$.

\section{Results}

In most of the conditions, there were no differences in results between the right and left hands and between the palm and back of the hands. ${ }^{4}$ Therefore, data combining these results are shown in Figure 9.

The results are very similar to those of Experiment 1. When the hands are at high levels, the results are consistent with the disembodied-eye phenomenon: right-left reversed on the forward-facing surfaces and objective on the backward-facing surfaces. In the lower three levels, the prevailing responses on the forward-facing surfaces are rotated and objective. On the backward-facing surfaces, the main responses are objective and up-down inversion. These results illustrate the same patterns as those of Experiment 1, except that the up-down inversion on the backward-facing surface was much greater.

The effect of height was statistically significant for both forward and backward-facing surfaces: The likelihood ra- tio chi-squares were 811.997 and 490.386 , respectively ( $d f=15, p<.001$, in each case). Table 2 shows the effect of height within each adjacent height-level pair. Although there were six levels of height, the effects of height were found only in the top-versus head-level and the head- versus chest-level pairs of both surfaces. This suggests that the head level is unique in our egocentric space. Also, it should be noted that the effect of height does not change continuously for hands, although it is nearly continuous for body surfaces.

There were some noticeable differences between Experiments 1 and 2. A clear difference was observed in the backward-facing conditions. In Experiment 1, up-down inversion was a minor response. It accounts for less than $15 \%$ of the responses on the back of the thighs and calves and was close to zero in all other conditions. In Experiment 2, however, the up-down inversion is more frequent for the backward-facing hands. It amounts to $35 \%$ of the responses in the levels lower than the head.

The difference between the body and the hand were statistically tested by the likelihood ratio test in each corresponding condition. In the backward-facing surfaces, significant differences were found in all but one (head) height levels: For the chest, trunk, and thigh levels, the likelihood ratio chi-squares were $72.351,67.083$, and 80.161 , respectively ( $d f=3, p<.001$, in each case). In the forward-facing surfaces, significant differences were found in the chest and the trunk levels (likelihood ratio chi-squares were 13.860 and 12.702 , respectively $[d f=3, p<.01])$.

\section{Discussion}

As mentioned before, up-down inversion on the back surfaces is interpreted as an outcome of the application of the bending-forward axes. Why do we do this for the hands more frequently than for the legs? It may be because of the movability of the hand. We can face our hand

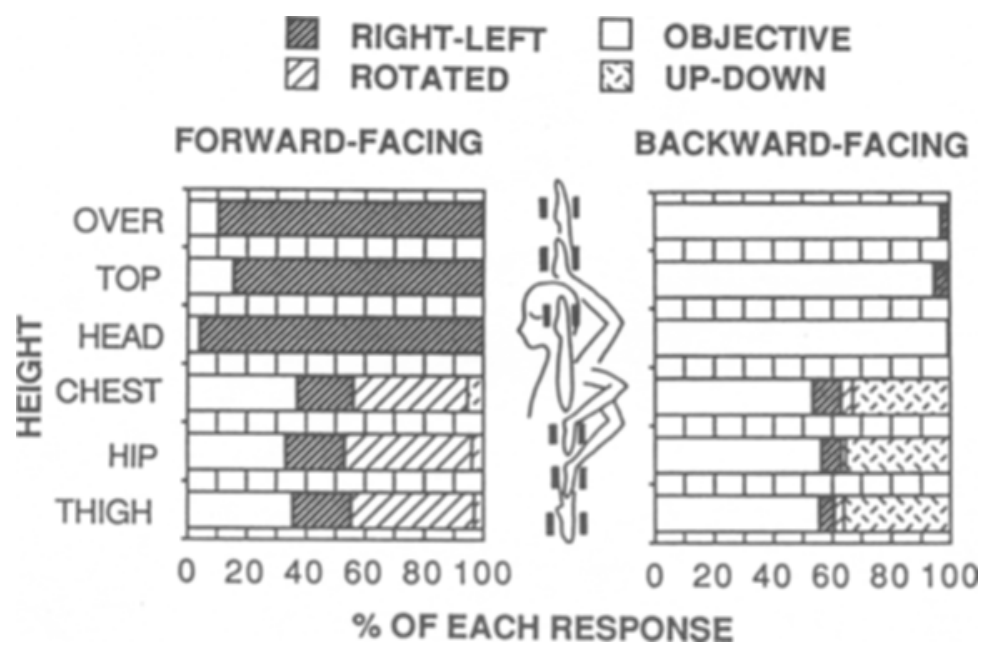

Figure 9. Mean rates of each response on the forward-facing and backward-facing surfaces of the hands at each height. 
Table 2

Percentage of Each Response in Each Condition and the Effect of the Surface Height in Each Adjacent Pair (Hand)

\begin{tabular}{|c|c|c|c|c|c|c|c|}
\hline \multirow[b]{2}{*}{ Conditions } & \multicolumn{4}{|c|}{ Response Category } & \multirow{2}{*}{$\begin{array}{l}\text { Likelihood } \\
\text { Ratio } \\
\text { Chi-Square }\end{array}$} & \multirow[b]{2}{*}{$d f$} & \multirow[b]{2}{*}{$p$} \\
\hline & OBJ & L-R & ROT & U-D & & & \\
\hline \multicolumn{8}{|c|}{ Forward } \\
\hline $\begin{array}{l}\text { Over } \\
\text { Top } \\
\text { Head } \\
\text { Chest } \\
\text { Hip } \\
\text { Thigh }\end{array}$ & $\begin{array}{l}16.7 \\
20.4 \\
11.2 \\
37.8 \\
32.9 \\
34.6\end{array}$ & $\begin{array}{l}83.3 \\
79.2 \\
88.8 \\
20.2 \\
20.0 \\
20.0\end{array}$ & $\begin{array}{c}0 \\
0 \\
0 \\
40.3 \\
42.5 \\
41.2\end{array}$ & $\begin{array}{c}0 \\
0.4 \\
0 \\
1.7 \\
4.6 \\
4.2\end{array}$ & $\begin{array}{r}2.55 \\
9.16 \\
284.54 \\
4.11 \\
0.191\end{array}$ & $\begin{array}{l}2 \\
2 \\
3 \\
3 \\
3\end{array}$ & $\begin{array}{l}.279 \\
.013 \\
.000 \\
.249 \\
.979\end{array}$ \\
\hline \multicolumn{8}{|c|}{ Backward } \\
\hline $\begin{array}{l}\text { Over } \\
\text { Top } \\
\text { Head } \\
\text { Chest } \\
\text { Hip } \\
\text { Thigh }\end{array}$ & $\begin{array}{l}96.7 \\
95.0 \\
99.2 \\
52.5 \\
55.8 \\
55.4\end{array}$ & $\begin{array}{r}3.3 \\
5.0 \\
0.8 \\
10.4 \\
7.5 \\
6.2\end{array}$ & $\begin{array}{c}0 \\
0 \\
0 \\
4.2 \\
1.7 \\
2.5\end{array}$ & $\begin{array}{c}0 \\
0 \\
0 \\
32.9 \\
35.0 \\
35.8\end{array}$ & $\begin{array}{r}0.84 \\
8.13 \\
181.57 \\
4.20 \\
0.70\end{array}$ & $\begin{array}{l}1 \\
1 \\
3 \\
3 \\
3\end{array}$ & $\begin{array}{l}.359 \\
.004 \\
.000 \\
.241 \\
.872\end{array}$ \\
\hline
\end{tabular}

Note-OBJ = objective; L-R = left-right; ROT = rotated; U-D = up-down.

not only by bending the neck but also by raising the hand. In both cases, as illustrated in Figure 10, the relationship between the head and the hand can cause the up-down inverted response on the opposite surface of the hand. With these two possibilities of underlying processes, the hands can give rise to more frequent up-down reversals. A leg, however, cannot be raised as easily as a hand. In consequence, up-down inversion on the thigh is less frequent than on the hands at the thigh level. It seems that the choice of frames of reference reflects the fact that the hand is the most movable part of the body.

The results of Experiment 2 revealed that the frames of reference found in Experiment 1 function in a similar way for both the body and the hand positioned alongside the body. It was also found that there were some quantitative differences between the thigh and the thigh-level hand, which indicates a need for an additional explanation of mental hand movements. These results also suggest that the body's movability is represented in the frames of reference.

\section{GENERAL DISCUSSION}

\section{Two Egocentric Frames of Reference}

To orient a two-dimensional tactile pattern, it is necessary for the pattern to be assigned a right-left and a top-bottom axis. The assignment of a set of axes is called a frame of reference. On the basis of experiments with four-way categorization, this paper proposed a notion of a bending-forward frame as one of the frames of reference for the lower part of the front and lateral surfaces (and limitedly for the lower part of back surfaces). This frame designates a set of egocentric axes in which the right-left axis is consistent with the head's right-left axis and the top-bottom axis is consistent with the top-bottom axis of the head in a bending-forward position. As depicted in Figure 4, this assignment of axes leads to the subject's right-left reversed and up-down inverted perception when the experimenter writes a letter on the front surface of the subject's thighs, because the right-left and the topbottom axes of the experimenter's frame and the subject's frame are both reversed. As a result, a $p$ is perceived as a d, which we have called a rotated response.

In contrast with this, the head and most back surfaces were firmly associated with another frame of reference that gives rise to the disembodied-eye phenomenon. This can be described by a frame of reference in which the right-left axis is consistent with the head's right-left axis and the top-bottom axis is consistent with the top-bottom axis of the head in an upright position. As a result, letters drawn on the forehead are perceived as right-left reversed, because the right-left axes of the experimenter's frame and the subject's frame are reversed when the two persons are facing each other. On the other hand, when the experimenter is behind the subject, the axes of the experimenter's frame and the subject's frame are consistent, resulting in an objective response by the subject. This axis description of the disembodied-eye phenomenon is not dissimilar to the "personal geography theory" that Corcoran (1977) proposed as an alternative to the "disembodied eye."

\section{Head-axes Hypothesis: Unifying the Two Frames}

An interesting question about these results is why we assign a top-bottom axis that is incongruent with gravity, as in, for example, bending forward while the head is upright and the eyes are closed. This could be a consequence of the fact that the head and its axes are generally of greater importance than the rest of the body. We can consider a "head-axes projection hypothesis": the axes of the head are projected onto the body surfaces that can be viewed directly within the actual range of possibilities of head and body movements. This explains bending-forward phenomena, namely the rotated responses on the lower front

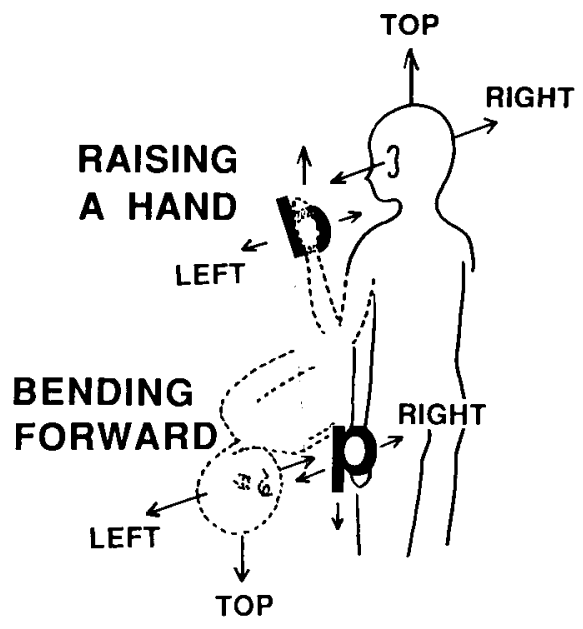

Figure 10. Two possible bases for the up-down inverted response on the backward-facing hands in low positions. 
and lateral surfaces and the up-down inverted responses on the lower back surfaces.

We can note, furthermore, that the disembodied-eye phenomenon is consistent with using the head axes without any projection; thus, we can consider a more general hypothesis that explains both classes of data, a "head-axes hypotheses." This gives much importance to the right-left and top-bottom axes of the head, projected within the constraints of body movement, as in the bending-forward frame, or without projection, as in the disembodied-eye phenomenon.

This sort of account can explain why there are the two functionally different regions in cutaneous perception on vertical body surfaces-the head as opposed to the rest of the body, as identified in Parsons and Shimojo (1987)as well as why the division is at the neck. The head axes can be projected only consistent with possibilities of head movement, so their projection can be applied only below the neck.

The usefulness of the head-axes hypothesis is not very clear if we investigate only head-level surfaces. Indeed, from this hypothesis, the right-left reversed perception on the forehead is only to be expected because the right-left axes of two facing persons (the subject and the experimenter) are reversed. If, however, we consider the data for the lower part of the body that suggested the bendingforward frame, the importance of the head axes becomes quite clear. A head-axes hypothesis implies a dynamic nature of the perceptual processes, such as the bendingforward frame (i.e., the projection of the head axes), as well as the importance of the head axes.

This paper began with a discussion of the disembodiedeye phenomenon. From the head-axes hypothesis that the present results lead to, however, the right-left reversed perception on the forehead should not be accounted for by a "disembodied eye." Rather, it should be explained by "embodied" head-axes, that is, axes that are intrinsic to the head but projectable within the possibility of body movements. The four-way categorization of the data used in the present paper (objective, right-left reversed, rotated, and up-down inverted) was necessary to arrive at this conclusion.

Although a concept of the head-axes projection is not dissimilar to that of the "pretend look" proposed by Corcoran (1977), there are at least two significant differences between the head-axes hypothesis and the pretendlook hypothesis: (1) The head-axes projection does not include the physically impossible body movements that the "pretend look" did, and (2) the head-axes hypothesis does not state a visual nature of the frame of reference, whereas the pretend-look hypothesis does. Rather, the former emphasizes a kinesthetic nature of the frame of reference, that is, head axes are applied to stimulated surfaces with or without projection, depending on possibilities of actual body movements. It does not necessarily mean that the stimulated surfaces are viewed by a kind of "eye." Therefore, the head-axes hypothesis is in accordance with a recent finding by Shimojo et al. (1989) that the congenitally blind exhibit the same response pat- terns as the sighted, suggesting a nonvisual nature for the disembodied-eye phenomenon.

\section{Further Issues}

There remains, however, the objective response on the front and lateral body surfaces below the neck that cannot be accounted for, because the head-axes hypothesis accounts only for the egocentric responses. In fact, an objective response is possible on all surfaces except the forehead. This exclusion shows that the head axes cannot be suppressed in the head region. Further exploration of the differences between the head and the rest of the body is needed for a complete understanding of cutaneous perception.

Another issue to be explored is whether the head-axes hypothesis can be applied to tactile stimuli other than symbols such as letters and numbers. Experiments using unknown shapes would be necessary to generalize the present hypothesis.

\section{REFERENCES}

AlLEN, D. B., \& RUdy, K. P. (1970). Perception of simple figures drawn upon the body surface. Perceptual \& Motor Skills, 30, 369-370.

Corballis, M. C., Zbrodoff, J., \& Roldan, C. E. (1976). What's up in mental rotation? Perception \& Psychophysics, 19, 525-530.

Corcoran, D. W. (1977). The phenomena of the disembodied eye or is it a matter of personal geography? Perception, 6, 247-253.

DUKE, J. D. (1966). Perception of finger drawings upon the body surface. Journal of General Psychology, 75, 305-314.

EVERITT, B. S. (1977). The analysis of contingency tables. London: Chapman and Hall.

holmes, D. S., Roeckelein, J. E., \& Olmstead, J. A. (1968). Determinants of tactual perception of finger-drawn symbols: Reappraisal. Perceptual \& Motor Skills, 27, 659-672.

Howard, I. P. (1982). Human visual orientation. New York: Wiley HowARD, I. P., \& TEMPLETon, W. B. (1966). Human spatial orientation. New York: Wiley.

KoffKA, K. (1935). Principles of gestalt psychology. New York: Harcourt-Brace.

Krech, D., \& Crutchfield, R. S. (1958). Elements of psychology New York: Knopf.

Natsoulas, T., \& Dubanoski, R. A. (1964). Inferring the locus and orientation of the perceiver from responses to stimulation of the skin. American Journal of Psychology, 77, 281-285.

Oldfield, S. R., \& PHillips, J. R. (1983). The spatial characteristics of tactile form perception. Perception, 12, 615-626.

Parsons, L. M., \& Shimojo, S. (1987). Perceived spatial organization of cutaneous patterns on surfaces of the human body in various positions. Journal of Experimental Psychology: Human Perception \& Performance, 13, 488-504.

Rock, I. (1956). The orientation of forms on the retina and in the environment. American Journal of Psychology, 69, 513-528.

Rock, 1. (1973). Orientation and form. New York: Academic Press.

Rock, I., \& HeImer, W. (1957). The effect of retinal and phenomenal orientation on the perception of form. American Journal of Psychology, 70, 493-511.

Sekiy ama, K. (1981, November). [Point of view in tactile perception: On the "reversal phenomena" (in Japanese)]. Paper presented at the 93rd Annual Conference of the Kansai Psychological Association. Kobe, Japan.

Sekiyama, K. (1982). Kinesthetic aspects of mental representations in the identification of left and right hands. Perception \& Psychophysics, 32, 89-95.

Shimojo, S. (1981). [On mental rotation experiments: A methodological study on imagery researches (in Japanese)]. Japanese Psychological Review, 24, 16-41 
Shimojo, S., Sasaki, M., Parsons, L. M., \& Tori, S. (1989). Mirror reversal by blind subjects in cutaneous perception and motor production of letters and numbers. Perception \& Psychophysics, 45, 145-152.

Witkin, H. A., Lewis, H. B., Hertzman, M., Machover, K., Meissner, P. B., \& WAPNER, S. (1972). Personality through perception. Westport, CI: Greenwood Press. (Original work published 1954)

\section{NOTES}

1. Recently, Shimojo, Sasaki, Parsons, and Torii (1989) reported that the congenitally blind exhibited the same response patterns as the sighted in similar cutaneous-perception experiments. Therefore, the eye metaphor, such as a "disembodied eye," should not be taken too literally.

2. The likelihood ratio tests were based on the cell frequencies, with 84 responses for each height condition ( 21 subjects $\times 4$ replications), using 5 (height) $\times 3$ (response category) contingency tables. The number of observed response categories were not four, but three, because there were no "up-down" responses on the front surface and no "rotated" responses on the back surface. The likelihood ratio test was used, although it is not totally appropriate for the comparison of these conditions from a within-subject-design experiment. Although efforts were made to extend the chi square tests to cases in which variables are not independent (beginning with Everitt, 1977), there is, as far as the author knows, no established method of such a kind.

3. This design does not separate hand orientation from body-relative height: The fingers pointed upward at the head level and above, and downward for the lower levels. It was assumed that finger direction does not influence perception of cutaneous patterns on vertically positioned hands-an assumption based on a preliminary experiment by the author, in which no significant differences could be found between finger-upward and finger-downward hands positioned vertically at shoulder-level. However, the direction of the fingers can be critical if the hands are positioned horizontally, as Parsons and Shimojo (1987) showed.

4. In the chest and hip levels of backward-facing conditions, however, the back of the right hand exhibited a trend such that the objective response was more frequent than that on the back of the left hand or on the palm of either hand. This trend (response-pattern differences among right-palm, right-back, left-palm, and left-back) was statistically significant in chest and hip levels: The likelihood ratio chi-squares for these two conditions were $41.300(d f=9, p<.001)$ and $18.05(d f=9$, $p<.05$ ), respectively.

(Manuscript received February 15, 1989; revision accepted for publication December 12, 1990.) 